\title{
Risk and reliability assessment of future power systems
}

\author{
W. A. Bukhsh \& K. R. W. Bell \\ Institute of Energy and Environment, University of Strathclyde, Glasgow, United Kingdom \\ T. Bedford \\ School of Management Sciences, University of Strathclyde, Glasgow, United Kingdom
}

ABSTRACT: Liberalisation of electricity markets, changing patterns in the generation and use of electricity, and new technologies are some of the factors that result in increased uncertainty about the future operating requirements of an electric power system. In this context, planning for future investments in a power system requires careful consideration of risk and reliability, and of the metrics with which these are measured. This paper highlights the need for consideration of a broader class of approaches to risk and reliability that have hitherto tended not to be an explicit part of the system development process in the electricity industry. We discuss a high level conceptual model that shows sources of uncertainty and modes of control for system operators and planners and offers a broad-brush approach to highlight risks at the planning stage. We argue that there is a need for new risk-informed criteria to help evaluate the necessary investments in electricity transmission systems. We further argue that the risk models that are developed for this purpose need to take better account of overall societal impact than is captured by traditional measures such as loss of load probability and loss of load expectation; societal impact should take account of frequencies of events with different levels of consequences, distinguishing, for example, between multiple small events and a single large event. This leads to discussion of a "disutility criterion" which has been previously studied in a health and safety context to distinguish between risk aversion and disaster aversion. This approach is new in the context of power systems.

\section{INTRODUCTION}

The structure and regulation of electricity industries has changed significantly over the last two decades. Most countries have restructured their power sectors to separate (to varying degrees) the activities of generation, network management and energy retail. Furthermore, new weather-dependent renewable generation has brought about challenges and many open questions, especially regarding the affect of these changes on the risks related to the reliability of the future electrical network.

Reliability standards for electricity networks are traditionally based on a deterministic criterion commonly known as ' $\mathrm{N}-1$ ' (Kirschen 2003, CIGRE WG $\mathrm{C} 1.17$ 2010). This is a rule under which elements in operation after a single unplanned outage must be capable of accommodating the new operational situation without violating operational limits (with the help of timely and appropriate operator action). This deterministic criterion is used to achieve an adequate level of reliability (ALR) in a power system.

\subsection{Transmission network utilities' activities}

Historically, the system operator (SO) has been mainly responsible for ensuring that the ALR is achieved. In the modern context of an electricity market, the SO should also facilitate competition between producers of electrical energy and procure services from different providers on both the generation and demand sides that allow the system to be operated, and should do so in a free and transparent way that minimises overall costs.
A transmission owner (TO, which, in some countries, is integrated with the SO within a 'transmission system operator' or TSO) is responsible for investing in and maintaining the assets of a power system. Thus, it is very important to think of reliability across all activities of an SO, TO or TSO: system operation, operational planning, asset management and system development, the last of which is concerned with the provision of sufficient facilities on the network such that it can be operated in accordance with relevant operating criteria.

Figure 1 shows the activities of transmission utilities across different time horizons. In the shorter time scales, e.g. day-ahead or on the day operation, the objective of a power system operator is to minimise total operational costs while maintaining an ALR. The main uncertainties include: forced outages of generation plant and network equipment; variations in weather that affect the power available from renewable generation and the demand for power; and market driven exchanges of power between areas. In these timescales, the values of many of these variables (demand, weather etc.) can be predicted with reasonable accuracy and, if the system has been sufficiently well planned, can be managed in real time operation. However, further out from real-time, uncertainties regarding planned outages of equipment to effect maintenance or the repair of defects must also be taken into account and weather and market behaviour are known with less certainty.

With many expected changes to future patterns of generation and consumption, and the development of a 'smarter' grid that increases the utilisation of a 
Invest in new facilities or take risk of higher Do maintenance and deplete network capacity maintenance and unreliability cost? or take risk of needing to do emergency repair?

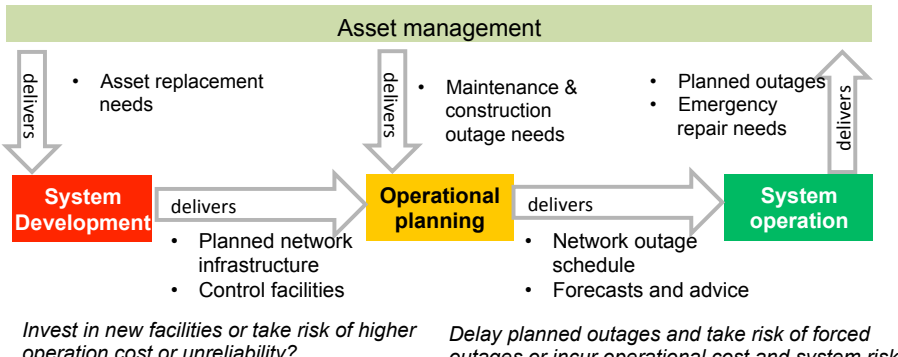

Invest in new facilities or take risk of higher Delay planned outages and take risk of forced

Figure 1: Activities of a TSO across the three time horizons

given set of power system assets through increased use corrective actions following disturbances and of flexible demand and more active management of distribution networks, there is a common view that the ' $\mathrm{N}-1$ ' criterion requires revision. From a risk perspective, it may seem odd that there is often no explicit reference to probability in the definition of 'N-1'. However, in the Security and Quality of Supply Standard (NETS SQSS 2014) applied in Great Britain (GB), for example, not all single element outages are 'secured against' such that there are no immediate adverse consequences: certain, less common but still 'credible' events, e.g. loss of a double circuit, are permitted to lead to a limited amount of demand disconnection. Moreover, "if there are adverse conditions such that the likelihood of a double circuit overhead line fault is significantly higher than normal", the system operator should configure the system in order that the loss of demand consequential to such a fault is less than normal. However, no guidance is given on the meaning of "significantly higher than normal". Furthermore, the ' $\mathrm{N}-1$ ' criterion may be non-conservative in some scenarios due to many potential dependencies between events, usually neglected, and in other situations may be overly conservative. Finally, although standards like those in GB quantify impact in terms of overloading of equipment, voltages or system frequency being outwith limits, poor damping of oscillations following disturbances or one or more generating units losing synchronism from the rest, the final impact on electricity users is often expressed only in terms of the initial power consumption that is interrupted. This fails to take into account the overall societal impact especially for events in which the amount of demand affected are large.

\subsection{Contributions of this paper}

This paper provides insights and guidance to those areas of power system planning where reliability goals can be more effectively achieved. It identifies several categories of uncertainties affecting the system that the system planner has to design, and we discuss a conceptual model depicting these uncertainties. We review standard risk measures that are used in power system planning problems and argue that such risk measures need to adapt depending on the type and location of outages, and that the strategy of 'one size fits all' is no longer appropriate in an evolving power system.

We briefly describe how changes in generation and demand patterns in the power sector are forcing a reconsideration of the deterministic criterion that is used to achieve an ALR and how researchers in at least one project, the GARPUR project, are asking whether advances in computational capability can enable a move away from a deterministic criterion to a probabilistic risk-aware criterion.

In conclusion, the following are the main contributions of this paper:

- a discussion of key drivers of reliability in power systems and related actions of power system operators;

- a discussion on measurement of risk in the context of power system planning;

- exposition of the need for risk models that adequately capture the overall societal impact, especially for events with large impacts.

The rest of the paper is organized as follows. Section 2 gives an introduction to the GARPUR project; section 3 briefly describes the system development process and the main drivers for investment. Section 4 discusses common risk measures that are used to assess the reliability of a power system. Section 5 reflects on the various uncertainties in power system planning and operation and proposes new ways in which risk in a power system could be quantified. Section 6 concludes the paper.

\section{THE GARPUR PROJECT}

The authors of this paper are participants in a European FP7 project called GARPUR: Generally Accepted Reliability Principle with Uncertainty modelling and through probabilistic Risk modelling (GARPUR 2013). The project is concerned with an improved understanding of risk attributes impacting power system reliability performance and corresponding metrics that, with new analytical tools, can lead to new criteria that can be used by system operators, asset managers and system developers to improve reliability performance and reduce cost.

The 4-year GARPUR project was launched in September 2013 and consists of 7 TSOs across Europe and 12 research and innovation partners. One key deliverable to date has been a formalisation of the way in which possible disturbances or unplanned events - 'contingencies' - are identified which the system operator should take into account when operating the system, i.e. should be 'secured' against. This is concerned with estimation of the 'residual risk' associated with the disturbances or variations that are not taken into account, i.e. are disregarded by the system operator (Karangelos \& Wehenkel, 
2016). Within this description, the ' $\mathrm{N}-1$ ' criterion secures against all single element outages but disregards all other variations. An explicit consideration of the residual risk opens the way to adaptation of the set of secured events in accordance with changing background conditions, e.g. changing probabilities of faults or short-term variations of output from weather-dependent renewables.

The authors of this paper are primarily involved in the system development part of the project Work Package 4 (WP4) - and hence the focus of this paper is on risk from the planning perspective. WP4 is concerned with an assessment of the risks that planners need to consider in the system development process. Network planners need to deliver a future grid that can be managed resiliently, and currently can only use planning tools that give them partial insight into the operation of, and demands upon, the future grid. There is a need for the development of new risk metrics that are fairly simple, yet still broadly reflect the societal impact of large scale outages and hence allow for an improved assessment of overall risk that can help inform future investment decisions.

\section{SYSTEM DEVELOPMENT PROCESS IN ELECTRICTY NETWORKS}

The general role of a system development planner is to ensure that sufficient facilities are installed on the system to enable it to be operated in accordance with relevant operating standards. In particular, the system development planner should ensure that the system's capability to transfer power from producer to consumer is sufficient. Because any insufficiency may require investment in the network's facilities and such investments take time, the system development process is commonly identified with timescales of a year or more ahead of real time operation, and therefore it is often described as a long-term planning problem.

\subsection{Components of power network costs}

The total cost of a power network may be described as consisting of the following parts (Bell 2015):

- the cost of network infrastructure and the cost of maintaining it $(I)$;

- the cost of operating the system $(O)$;

- the cost to consumers of unreliability of electricity supply $(X)$

The goal of a system planner is to minimise the overall expected cost $(I+O+X)$ over some given period of time. In the time-scales in which investment in network infrastructure takes place, the planner must deliver a network that can be operated reliably, and must be mindful of future $O+X$. A relative lack of investment in network infrastructure would lead either to greater $O$ (the wholesale elec- tricity market's access to its preferred generation is restricted which implies that other, higher cost generation should run) or greater $X$ (some additional loss of supply takes place from time to time which has some economic and social impact). Clearly, in quantifying the anticipated future values of $O$ and $X$ for a given network infrastructure, account must be taken of the way in which the system is operated. This includes use of new control facilities such as special protection schemes, demand side management or actively managed distribution.

Figure 2 shows that overall cost will generally increase as the reliability of a system is increased (Wang \& Singh 2009). However, below a certain value of reliability index, the increasing costs of unreliability dominate. Total cost is at a minimum at $\mathrm{R}^{*}$, which determines the optimal level of reliability.

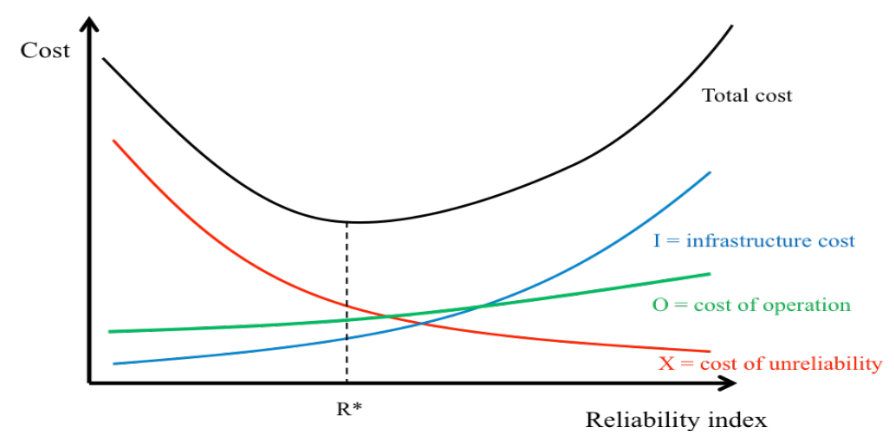

Figure 2: A graph showing relationship between reliability and overall cost of power system.

The uncertainties that affect system development are greater than those that apply, for example, in system operation. Thus, a key objective for a system planner is to identify efficient improvements to the system development process that provide benefits in terms of better management of risk without imposing an excessive burden on business processes and analysts. The balance sought should make more explicit use of probabilistic information on operating conditions and disturbances than is common at present and use appropriate metrics to:

- identify how much modelling is required to give sufficient confidence with respect to assessment of future operation of the system for a given generation capacity/demand/market scenario;

- make use of information on system performance from a set of scenarios to identify and justify any investments in new network facilities that are required.

\subsection{The two drivers of investments in transmission}

In respect of facilitating system operation in accordance with operating standards, the following two drivers may be noted:

- provision of power transfer capability such that the demand for electricity in a given area can be met with a given, minimum level of reliability, i.e. reliability driven capability; 
- provision of power transfer capability such that demand for electricity on the system can be met, over the medium to long term, in an economically efficient manner, i.e. economics driven capability.

Economics driven investment involves an assessment of relative costs and benefits of investment in enhancement of power transfer capability compared with network congestion and running of expensive, 'out of merit' generation.

\section{COMMON METRICS USED TO EVALUTE RELIABILITY OF POWER SYSTEMS}

Due to the integrated nature of power systems, it is possible for failures in any part of the system to cause severe disruptions. These disruptions can be very costly to the electricity market and society. Ideally, supply should be made continuously to customers but that is expensive and arguably not feasible. Due to the random nature of failure, it is accepted that any real-world system will have a non-zero risk of suffering power outages. The risk can be reduced, for example, by installing more reliable equipment, having more redundancy on the network or by providing additional capacity as generation reserves, but always at additional cost. Given the complexities of power systems, the trade-off between costs and benefits of different interventions commonly depends on particular metrics, outlined below.

\subsection{The capacity margin}

The capacity margin is the proportion by which the total expected available electricity generation exceeds the maximum expected level of demand at the time at which that demand occurs (NETS SQSS 2012). A positive capacity margin acts as an insurance against occasional unexpected losses of generation or increases in demand and is normally expressed as the percentage calculated by:

$M_{C}=\frac{G_{C}-D_{\text {peak }}}{D_{\text {peak }}} \times 100$

where $M_{C}$ denotes the capacity margin, $G_{C}$ is the total capacity of generation installed on the system and $D_{\text {peak }}$ is the annual peak value of total demand.

The capacity margin has been used to give an indication of how much generation capacity should be built in order to have a certain level of reliability of supply. However, it does not take into account the limitations of the network and does not distinguish between different types of generation, e.g. thermal, hydro and wind that have very different average available power. Transfers of power on interconnectors, i.e. transmission connections between different countries, should also be taken into account.

\subsection{The derated capacity margin}

As not all generation capacity will have the same availability or always be able run at its theoretical maximum, in order to remove some of the limitations in the measure of capacity margin, sometimes de-rated capacities are used when calculating a derated capacity margin. The British regulator for gas and electricity, Ofgem, lists de-rated capacity factors for different type of generators in their capacity assessment reports (Ofgem 2013).

\subsection{Loss of load probability}

Loss of load probability (LOLP) is another common measure that is used to evaluate reliability of power system and is defined as the probability of the system demand exceeding the available generation. The LOLP is usually calculated for each year using the annual peak demand. However, it is sometimes quoted for each day using the peak demand of the day or on an hourly basis using the peak demand of each hour. It characterizes the adequacy of generation to meet the total expected peak demand. Mathematically, LOLP can be defined as follows:

$L O L P=P\left(D_{\text {peak }}>A_{G}\right)$

where $D_{\text {peak }}$ is total peak demand of the system and $A_{G}$ is the total power generation available at the time of the peak.

\subsection{Loss of load expectation}

Loss of load expectation (LOLE) represents the number of hours per annum in which, over the longterm, it is statistically expected that supply will not meet demand (Billinton \& Allan 1996). Mathematically, LOLE is defined as follows:

$L O L E=L O L P \times T$

where $T$ is the time period. Usually $T$ is taken as 8760 hours and $L O L P$ as the loss of load probability for each hour that models the loss of load expectation in hours per year. As part of the Electricity Market Reform in Great Britain, the UK Government has set a reliability standard of 3 hours LOLE per year (Ofgem 2013), used to identify the amount of generation capacity to be bought in a 2 or 3 -year ahead capacity market. In practice, the standard used in the GB capacity market reflects not the number of hours in which interruptions to supply to electricity users might be expected but the number of hours in which the system operator might be expected to take emergency actions to address a shortfall of available generation relative to demand. Disconnection of some amount of demand is among the possible actions but is only a last resort after using contracted demand side reductions, decreases of interconnector exports, increases in interconnector imports, 
'maxgen' (the ability of some generators to exceed their normal rating for a short period of time), and demand reduction through a decrease in the voltage supplied to electricity users through the distribution networks (Bell 2014).

\subsection{Expected energy not supplied}

A final metric that is in common use takes account of both the initial power interruption and the time taken to restore supply in a quantification of the total energy that is not supplied to electricity users. It is known as expected energy not supplied (EENS) and is calculated as follows (Wang 2008):

$E E N S=8,760 \times \sum_{C_{i} \neq 0} C_{i} \times L O L P_{i}$

where $C_{i}$ is the amount of energy curtailed and $L O L P_{i}$ is the corresponding loss of load probability .

What the Great Britain system operator National Grid reports as a 'reliability' of $99.99999 \%$ for the year 2014/15 (National Grid 2014) is best understood as the energy supplied after the occurrence of faults originating on the transmission system, expressed relative to the total energy that would have been supplied had the transmission faults not occurred. One limitation of the metric is the necessity to estimate the total energy that would have been supplied. (Due to the occurrence of the most frequent cause of interruptions to supply, i.e. faults on the distribution network, the overall reliability of supply for the GB power system as a whole is less than the National Grid figure).

\subsection{Limitations of the existing reliability measures}

The problem with the reliability measures described here is that they indicate problems in the system but do not indicate the severity of each problem and therefore are not very meaningful. For example, they do not take into account whether there were many short periods of lost load or a few long periods. Second, they do not take account of how much load was lost each time. Third, they do not look at any follow on consequences. Further, in calculations of these metrics, it is commonly assumed that data about demand and generation capacity are perfectly accurate and that the generation on a system is infinitely flexible (i.e., generation can ramp-up or rampdown freely and rapidly) or that the system operator always has so much notice of a potential shortfall of generation relative to demand that it can dispatch additional available generation in sufficient time. Such assumptions are not true in reality.

\section{MANAGEMENT OF RISK}

From the perspective of a system planner, a system design choice will turn out poorly if:
1. in its planned-for state, the system suffers from interruptions to supply more frequently or with greater size than was expected; or

2. in its planned-for state, the system frequently operates at a cost above the theoretical expected optimum; or

3. changes in demand and/or generation are not anticipated in sufficient time to create the appropriate infrastructure, leading to unplanned interruptions to supply and/or higher costs.

The first two of these consequences are the results of aleatory uncertainties regarding demand, infrastructure state and environmental conditions, which impact on the ability of the operator to manage the system. The third point relates to state of knowledge uncertainty about the future development of the overall system. A further state of knowledge uncertainty arises from approximations used in modelling system responses to disturbances.

These risks are mitigated in practice in a number of ways. Most obviously the system operator takes actions to ensure that the system stays within limits - this may involve allowing, for example, thermal loads on lines to temporarily exceed the usual norms (defined in terms of continuous loading), or moderate rises in voltages may be tolerated for a short time. In the case of excess costs of operation, the main course of mitigation is investment to reduce overall costs in the longer term. This might involve new, cheaper generation or additional network capacity to reduce congestion. However, in a liberalised electricity industry, investment in generation capacity is not within the scope of the network planner, though planners play an important role in signalling potential issues and opportunities.

Taking account of the risks which are mitigated, the system planner is mainly concerned with the residual technical risks, either arising in the plannedfor state, or arising from changes that were reasonably foreseeable. In order to assess these risks, we need to be able to model rates at which the system deviates from its desired performance despite reasonable interventions of the system operator.

In the context of risk management, risk models refer to the use of quantitative or statistical methods to determine the aggregate risk based on a set of foreseeable future operating conditions. Risk assessment is an essential tool for a system planner to identify potential intervention where the most significant lowering of risks can be achieved. The objective of managing risk is to decrease the probability of events that have an adverse impact, decrease their impact, or both. For this reason, probability models are required to take account of conditional probabilities among various factors that influence the operating conditions of a power system. Further, in order to build an adequate risk model, it is important to take account of the overall societal impact. 


\subsection{Uncertainty in future operating conditions}

In order to quantify the impact of an event, it is necessary to know the initial conditions of a system, i.e. its initial operating state, and the available corrective actions. In the context of a power system, this means the location and levels of demand and generation, the network topology, available corrective controls and also the reliability criterion, e.g. ' $\mathrm{N}-1$ ', used by the system operator to decide the operating state.

The system planner should anticipate a range of conditions that might be faced by the system operator and whether they are likely to have sufficient facilities available to allow those conditions to be managed in accordance with the prevailing reliability criterion.

The functional dependence of an operating state of a power system can be characterized by endogenous and exogenous variables. The bottom-most boxes in Figure 3 are exogenous variables while the rest are endogenous. The planned outages box in Figure 3 refers to maintenance of transmission and generation equipment. The planned outages that might be allowed depend on the expected operating state of the system; the operational planner can move them forward or backward in time depending on the expected operating conditions and various other factors, whilst maintaining a reliable system. Unplanned outages refer to the random events that cause outages of generation and/or transmission components. In Figure 3 this is shown by a dotted line because a system operator has no control over their timing or location.

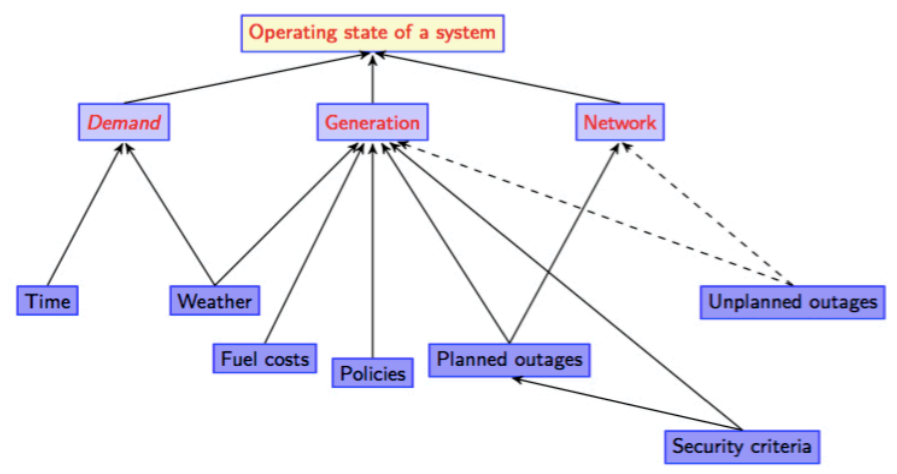

Figure 3: Components of an operating state.

It can be seen from Figure 3 that demand for power depends on the weather and, because demand has diurnal and seasonal dependencies, the time of the day. Similarly, generation from wind turbines and solar panels depend largely on the weather. For system planning studies and in system operation, it is very important to take proper account of temporal and spatial correlations between variables and of wholesale market preferences for how demand is to be met.

Various models of power system responses to disturbances exist, e.g. load flow, which are used by the planners. However, such models need to be used within a broader set of scenarios which are designed to explore the conditional risk levels in settings which can easily arise. System planners use these tools to evaluate different options for investment in new facilities. With the need to model so many different situations, a range of tools is used in practice with different levels of precision in order to refine the set of options under consideration. Finally, a decision must be made on whether to invest and in which options to do so. Although different frameworks for decision making have been proposed, e.g. min-max regret as discussed in (Fang \& Hill 2003) or a multi-objective approach as in (Karimi \& Ebrahimi 2015), suitable metrics of the risks associated with different events are still required.

\subsection{Measurement of risk}

Historically, power system planners have managed risk by setting thresholds and safety margins at levels determined by industry experience, e.g. the ' $\mathrm{N}-1$ ' rule. Although attempts have been made to quantify a 'value of lost load' or 'customer damage functions' (see, for example, Kariuki and Allan 1996 and de Nooij 2007), because of the complexity of assessing the economic and social impact of outages, not least the difficulty of modelling the time and duration of outages, there is - as yet - no overall formulaic approach that optimises the expected economic and social benefits of investment in the network against the investment costs. Such an approach would in any case be far too burden-some for the optioneering stages of the system design process. An alternative is to look for risk heuristics which broadly reflect what the societal impact would be of different kinds of outages.

It is worth making an analogy with risk tolerance concepts used in Health and Safety (H\&S) settings. Figure 4 shows three regions of risk tolerance. In a $\mathrm{H} \& \mathrm{~S}$ context the upper line is the limit of tolerable risk. Risks above that level are not acceptable and will not be traded against the cost. Below the threshold line is the tolerable region, commonly known as the As Low As Reasonably Practicable (ALARP) region. The risk of an event in ALARP is tolerable only if risk reduction is impracticable or its cost is disproportionate to the improvement gained. The region below the solid line at the bottom is the broadly acceptable region because either the probability of an event is negligibly small or the impact is very small. In a safety context, risks above the upper line are not tolerated, risks below the lower line do not have to be considered in trade off analyses (though this does not remove the general obligation to follow good practice), and risk in the ALARP region should be managed through trade-off studies. The analogies with power system planning are not exact, but, for example, there is an upper limit of 3 hours LOLE for insufficiency of generation in the GB system (Ofgem 2013). The system planner will not be con- 


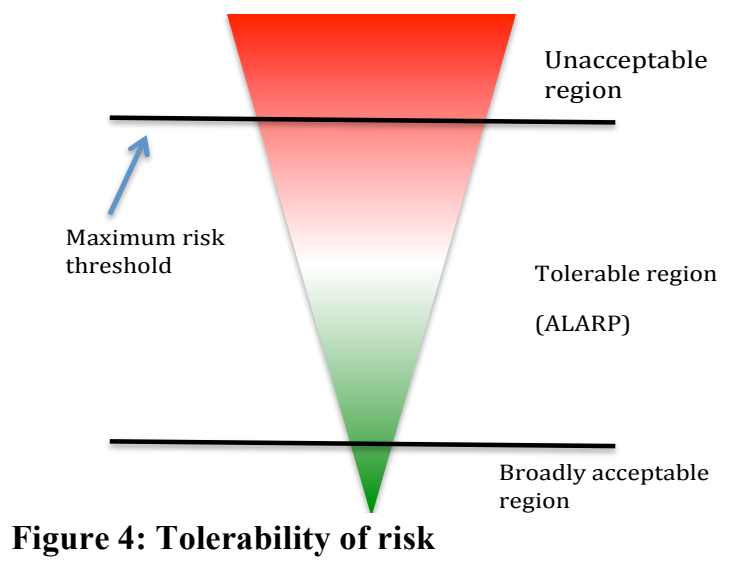

cerned with minor outages that should be dealt with by normal good operational practice, and hence there is an analogy to the lower limit. The system planner is mainly concerned with cost effectively dealing with risk in the middle region.

In a power system context, there is the risk of cascading outages that can follow a significant disturbance and can lead very rapidly to a large-scale blackout from which recovery is technically challenging and time-consuming (IEEE 2007). Moreover, the impact to society of complete loss of power - loss of communications, heating, cooling, lighting, electric transport, water and sewage pumping, traffic management systems, etc. - is very much larger than partial loss of power (Southwell 2014). For this reason, it is very important to distinguish the risks between many small disturbances and a single infrequent large disturbance.

\subsection{Resilience and disutility}

'Resilience' of a system can defined as its ability to recovery from shocks or challenges. Societal resilience against many small outages, but lack of resilience to large ones suggests that a non-linear weighting can provide a reasonable and practical approach to measuring risk. Similar challenges exist in Health and Safety risk measurement. Indeed, there is a well-established literature that looks at FN curves and criteria lines, and at risk measures that give a non-linear weighting to larger sizes of accidents.

Wilson (Wilson 1975) and Okrent (Okrent 1981) introduced a criterion that could be used to distinguish between one single large event and many small events. Okrent considered how to measure the overall risk posed by continuing use of an industrial installation or system, in which a sequence of accidents with differing numbers of fatalities might (in principle) occur throughout its operating life.

The criterion proposed by Wilson and Okrent is called a 'disutility criterion' and is effectively a summary statistic for group risk. The disutility $d$ is defined as:

$$
d=\sum_{n \geq 1} f_{n} \times n^{\alpha}
$$

where $f_{n}$ is the annual frequency of accidents involving $n$ fatalities and $\alpha$ is a risk aversion factor. When $\alpha=1$, this is simply the expected number of fatali- ties per year. Okrent suggested that 1.4 was a reasonable choice reflecting societal aversion to large accidents.

A more recent treatment is given by Bedford (Bedford 2013) who showed that the approach suggested by Okrent, despite its attractions, did not do what Okrent was seeking to achieve, and provided a broader framework in which both disaster aversion (i.e., aversion to large accidents) and uncertainty aversion (i.e., unwillingness to gamble) could both be modelled. As Bedford pointed out, the formula is not really risk averse (as Okrent described it) when $\alpha$ $>1$ but is actually disaster averse. Since there are often large uncertainties about the frequency of large fatality accidents it is of interest to understand how to modify the calculation to reflect risk aversion to uncertainty in the rate. Bedford shows how this can be done, giving, for example, a two parameter family where one parameter controls disaster aversion (our aversion to large accidents) and the other controls risk aversion (our aversion to uncertainty about the rate of incidents of any kind).

Similar concepts would seem to be applicable also in the context of risk measurement for power systems. The impacts of very large outages are enormous and difficult to quantify because of the difficulty of identifying and assessing all the knock-on consequences. This suggests that large outages should be weighted non-linearly more than small outages which have a less disruptive effect on society. However, the down-side of using a power law as suggested by Okrent is that the measure becomes very sensitive to uncertainties about the frequencies of very low frequency events. By definition, these are very difficult to credibly assess. Furthermore, however, one could argue that society is similarly averse to all very large outages, suggesting that outage size impact grows less strongly once in the class of very large outages. Also, the measures that can be taken to reduce the probabilities of different very large outages are the same, so differentiation between sizes of very large outages does not necessarily help in determining optimal strategies. This suggests using a disutility function that has a power law with $\alpha>1$ for low to large size outages but which then switches to a different power law behaviour with $\alpha<1$ for large to very large outages. The size of the outage may be measured in terms of the number of consumers losing power or the total load initially lost. Appropriate values of $\alpha$ can be determined by risk analysts through the use of standard preference questions with, for example, system planners to assess their implied preferences.

The use of a simple risk metric like this can be used in conjunction with a simulation system based on the factors set out in Figure 3 to provide summary feed-back to system planners in the optioneering and assessment phases of system planning. Furthermore, the use of two classes of outage and 
recognition of disaster aversion can help to identify and justify measures to reduce the size of a major disturbance even if its probability of occurrence cannot be quantified with any confidence or reduced significantly. The set of measures that a power system planner can consider forms a 'defence plan' and includes such interventions as automatic underfrequency load shedding and planned system islanding (CIGRE WG C1.17 2009).

\section{CONCLUSIONS}

The purpose of power system development planning is to enable future operation of the system in accordance with appropriate system operation rules or conventions. One such convention that has been commonly used in power systems to date is the ' $\mathrm{N}-1$ ' criterion that states that system limits should still be respected even after the unplanned outage of any one element of the system. Proper consideration of various uncertainties and risks to system operation is an important part of the business case for any system development plan. The different impacts that different disturbances on a power system might have been discussed. The worst case is that supply of power to electricity users is interrupted without their consent. A number of risk metrics commonly used in power system planning and operation have been described and argued to be inadequate in respect of capturing the differences between large and small interruptions to supply of power, and long and short duration outages. It has been further argued these differences are highly material in terms of societal impact and the duration and technical difficulty of recovery.

As a consequence of the above, we propose the construction of risk models that take better account of the overall societal impact than simple loss of load metrics do within many electricity transmission network utilities currently. The societal impact should take account of frequencies of events with different levels of consequences, for example between multiple small events and a single large event. Due to the difficulty of modelling the size and duration of outages, we also propose the use of a "disutility criterion" based on quantities that can be quite readily modelled by system planners and used as a proxy for impact that reflects 'disaster aversion'. However, because such a metric can be very sensitive to assumed frequencies of events, we also suggest an approach which primarily sensitive to the difference between small and large events, with less emphasis on the differences between alternative very large events (which are inherently highly uncertain in magnitude but all highly disruptive).

\section{ACKNOWLEDGEMENT}

The research leading to these results has received funding from the European Union Seventh Framework Programme under the project titled GARPUR, Grant Agreement No 608540.

\section{REFERENCES}

Bedford, T. 2013. "Decision making for group risk reduction: dealing with epistemic uncertainty." Risk Analysis.

Bell, K. 2014. "Electricity Capacity Assessment 2014: Response to consultation on methodology".

Bell, K. 2010. "Methods and Tools for Planning the Future Power System: Issues and Priorities." The Institution of Engineering and Technology.

Billinton, Roy and Allan, Ronald N. 1996. Reliability Evaluation of Power Systems. New York: Plenum Press.

CIGRE WG C1.17 2010. Planning to Manage Power Interruption Events. Technical Brochure 433, CIGRE, Paris.

de Nooija, Michiel, Koopmansb, Carl and Bijvoet, Carlijn. 2007. "The value of supply security: The costs of power interruptions: Economic input for damage reduction and investment in networks." Energy Economics.

Fang, R. and D. J. Hill, D.J. 2003. "A new strategy for transmission expansion in competitive electricity markets." IEEE Transactions on Power Systems.

GARPUR 2013. "Generally accepted reliability principle with uncertainty modelling and through probabilistic risk assessment".

IEEE Task Force, 2007 Blackout Experiences and Lessons, best Practices for System Dynamic Performance, and the Role of New Technologies, Final Report. IEEE.

Karangelos, E. and Wehenkel, L. 2016 "Probabilistic Reliability Management Approach and Criteria for Power System Real-time operation." Proc 19th Power Systems Computation Conference.

Kariuki, K.K and R. N. Allan, R.N. 1996. "Evaluation of reliability worth and value of lost load." IEE Proceedings in Generation Transmission and Distribution 143, no. 2.

Kirschen, D.S, Bell, K.R.W. Nedic, D.P., Jayaweera, D. and Allan, R.N. 2003. "Computing the Value of Security", IEE Proceedings on Generation, Transmission and Distribution, vol. 150 , no. 6 .

National Grid 2014. Annual report and accounts. Technical Report.

NETS SQSS 2014. National electricty transmission system security and quality of supply standard, version 2.2.

Ofgem. 2013. Electricity capacity assessment report. Technical report, Ofgem.

Okrent, D. 1981. "Industrial risks." Proceedings of the Royal Society of London Series a-Mathematical Physical and Engineering Sciences.

Southwell, Phil. 2014 "Disaster Recovery within a CIGRE Strategic Framework: Network Resilience, Trends and Areas of Future Work." CIGRE, Paris.

Wang, L. \& C. Singh 2009. "A conceptual study on reliability constrained transmission system planning including wind power". Power \& Energy Society General Meeting, IEEE

Wang Xi-Fan, Song Yonghua and Irving, Malcolm 2008. Modern Power System Analysis. Springer. 